\title{
Receptive anal sex in women and risk of colorectal cancer (2009-20 I4): a retrospective analysis of NHANES
}

\begin{abstract}
Background: Risk factors for early presentation of colorectal cancer are a topic of particular current interest. The objective of the current study is to examine if there is an increased risk for colorectal cancer in women who engage in receptive anal intercourse as compared to patients who have not engaged in receptive anal sex.

Methods: The National Health and Nutrition Examination Survey (NHANES) was considered for women who answered the sexual health questionnaire (SXQ) during the survey cycles that included a SXQ item referring to anal sex (2009-2014). The questionnaire assessed sexual health behaviors including history of receptive anal intercourse in women. Additional NHANES data was matched for demographics, including race, as well as medical diagnoses such as colorectal cancer. Statistical analysis was conducted using the statistical software, $\mathrm{R}$.

Results: A total of 7,781 women completed the NHANES SXQ between 2009 and 2014 but only 6,596 answered the question mentioning receptive anal intercourse. A total of 2,203 women $(28.3 \%)$ stated that they had a history of receptive anal intercourse at least once. From 2009 to 2014 , there were a total of 19 participants $(0.29 \%)$ with a diagnosis of colorectal cancer, with 5 patients who had both a diagnosis of colorectal cancer and history of anal intercourse. The risk of a diagnosis of colorectal cancer with a history of receptive anal intercourse in women was 0.0023 , whereas the risk of a diagnosis of colorectal cancer with no history of receptive anal intercourse was 0.0032 . There was no significant evidence that receptive anal intercourse in women is associated with risk of colorectal cancer.

Conclusion: No significant difference was found in relative risk of diagnosis of colorectal cancer in women who engaged in anal intercourse as compared to those who did not engage in anal intercourse.
\end{abstract}

Keywords: colorectal cancer, anal intercourse, women
Volume 10 Issue 4 - 2019

\author{
Chethan Ramprasad, ' Vincent Major ME \\ M, ${ }^{2}$ Yian Zhang, ${ }^{3}$ Hannah LaBove, ${ }^{4}$ Shida \\ Haghighat, ${ }^{5}$ Ofer Fass,' Mariam El-Ashmawy' \\ 'Internal Medicine Residency, NYU Langone, USA \\ ${ }^{2}$ NYU Langone Health's Predictive Analytics Unit, USA \\ ${ }^{3}$ Department of Biostatistics, NYU Langone, USA \\ ${ }^{4}$ General Surgery Residency, University of Miami Miller School of \\ Medicine, USA \\ ${ }^{5}$ Department of Internal Medicine, Keck School of Medicine of \\ USC
}

Correspondence: Chethan Ramprasad MD, Department of Medicine, NYU Langone Health, 550 First Avenue, NY, NY 10016, Email chethanramprasad@gmail.com

Received: July 01, 2019 | Published: July 18, 2019

\section{Introduction}

Colorectal cancer (CRC) is the third most common cancer diagnosed in the United States and is also the third leading cause of cancer deaths in the United States. ${ }^{1}$ While there has been a decline in its overall incidence, presumably due to screening modalities, there has been an increase in the incidence of CRC diagnosed in younger individuals (age $<50) .{ }^{2}$ Since CRC has traditionally been a disease affecting individuals greater than age 50, the screening guidelines for low risk individuals accordingly target those in the $5^{\text {th }}$ decade of life. ${ }^{3}$

CRC diagnosed in younger individuals is thought to be more aggressive than when diagnosed after age 50 , in part because younger patients tend to present with advanced disease. ${ }^{3}$ CRC in individuals $<50$ is typically diagnosed during the work up for signs and symptoms associated with extensive disease. ${ }^{4}$ This is in contrast to older patients who tend to be diagnosed with early stage disease during screening colonoscopy.

Excluding genetic predisposition, there is a paucity of research regarding the risk factors associated with early onset CRC. Obesity is a recognized risk factor for $\mathrm{CRC}$, and the increasing prevalence of obesity among younger individuals is likely a contributing factor. ${ }^{5}$ However, accepting obesity as the primary driver of CRC among young patients is narrow in scope. It is also necessary to evaluate behavior and lifestyle choices that might explain the rising incidence among the younger population. Sexual activity tends to decrease

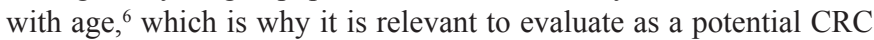
risk factor in the young. An area of particular interest is the possible association between the increasing practice of receptive anal sex and the rising incidence of early onset CRC. Around $10 \%$ of heterosexual couples have engaged in anal intercourse, albeit with varying frequency. ${ }^{7}$ Homosexual males partake in anal sex to an even greater degree, described to be six times more frequent than the occurrence in heterosexuals. ${ }^{8}$ Even so, heterosexual anal intercourse, especially among females, appears to be on the rise, with an estimated lifetime prevalence of $22 \%$. $^{9}$ However, it is difficult to know whether this finding is due to individuals feeling less stigmatized and thus more willing to report anal intercourse. ${ }^{9}$ Nevertheless, it has been suggested that the overall frequency of receptive anal intercourse is four times greater in women as compared to men who have sex with men due to the number of women who have engaged in the behavior. ${ }^{10}$

It has been suggested that the risk of colorectal cancer may be increased due to alterations in the microbiome. The microbiome of the female genital tract is altered after exposure to semen ${ }^{11}$ thus it would be plausible that receptive anal sex alters the rectal and colonic microbiota. Only one study has been conducted examining the effect of receptive anal sex on the rectal microbiota. Kelley et al. found that the stool microbiota of MSM (men who have sex with men) who engaged in receptive anal sex was enriched for Prevotella species, 
however this enrichment was not uniform across all MSM who engaged in receptive anal sex. ${ }^{12}$ Additional studies have demonstrated that Prevotella, along with Fusobacterium, Porphyromonas, and Bacteroidetes, was among the more prevalent microbiota in CRC patients. ${ }^{13-15}$ Despite a potentially increased prevalence of Prevotella in CRC patients, there is no evidence that demonstrates a causal relationship between receptive anal sex and the development of CRC due to the presence of Prevotella, or other species, in the microbiome.

The HPV virus can be transmitted via anal sex and is the leading cause of anal cancer. ${ }^{16}$ While the rectum and colon are in close proximity to the anus, the cellular makeup differs with the anus consisting of squamous epithelium and the colon and rectum consisting of columnar epithelium. There has been a known relationship between HPV and the malignant transformation of anal squamous cells, but recent studies have also suggested that the HPV virus could be involved in the pathogenesis of CRC and the malignant transformation of columnar cells. ${ }^{17-20}$ A study by Lee et al. ${ }^{20}$ found HPV-18, which is commonly found in anogenital cancers, to be frequently present in $\mathrm{CRC}$ tissue. This finding was statistically significant when compared to the presence of HPV in cancer-free colonic tissue. A study by Bernade-Dones et al. ${ }^{19}$ found a statistically significant prevalence of HPV-16 infection and its integration into CRC tissue among a population of Caribbean Hispanics with the disease. ${ }^{19}$ In contrast, a study by Vuitton et al..$^{21}$ did not find the presence of HPV in CRC tissue. All things considered, the association between CRC and HPV remains unclear.

There have been no studies to date that evaluate whether receptive anal sex is associated with CRC due to mechanisms independent of HPV. Individuals who engage in receptive anal sex have been shown to have a more diverse fecal microbiome, and this finding might be involved in the development of CRC. ${ }^{12}$ When participating in anal sex, there is frequent use of sexual lubricants, which are usually hyperosmolar solutions. Fuchs et al. found that these hyperosmolar lubricants caused injury to intestinal epithelium, most pronounced at $10 \mathrm{~cm}$ from the anal verge, ${ }^{22}$ and could also be a risk factor for CRC. With a greater understanding of the mechanism responsible for the increased frequency of CRC diagnosed in young individuals, we will be better equipped to manage and treat the disease. The goal of our study is to analyze whether there is an association between the increased incidence of early onset $\mathrm{CRC}$ and a history of receptive anal sex in women.

\section{Methods}

The NHANES (National Health and Nutrition Examination Survey) database was queried for men and women who answered the sexual health questionnaire (SXQ) across the United States during the survey cycles asking about anal sex (2009-2014). NHANES is a program of studies that is designed to assess health and nutritional status of the United States population through interview and surveys. Data is publically available and is nationally representative. Demographics, including gender and race, as well as medical diagnoses, specifically colorectal cancer, were extracted from the relevant NHANES questionnaires DEMO (demographic data) and MCQ (medical conditions). The entire analysis is limited to women because men with a history of receptive anal intercourse could not be reliably identified and self-reported HPV diagnosis was not ascertained for men. Question SXQ836 asked, "In your lifetime, with how many men have you had anal sex?" The question did not distinguish between receptive and penetrative anal sex. Similarly, SXQ809 asked "have you ever had sex with a man, including oral or anal?" and did not specify receptive anal sex versus oral sex.

Women with a history of receptive anal sex were identified from question SXQ 706, which directly asked "Have you ever had anal sex? This means contact between a man's penis and your anus or butt." Analysis was only included for participants who responded "yes" or "no". Those participants with "missing or "refused" responses were excluded.

Participants with a history of colorectal cancer were identified from question MCQ220 "Have you ever been told by a doctor or other health professional that you had cancer or malignancy of any kind?" and then subsequently MCQ230 "what kind of cancer was it?" to which participant's answers were categorized into 29 named groups, including colon (16) or rectum (31).

HPV data was identified from question SXQ753, which asked, "Has a doctor or other health care professional ever told you that you had human papillomavirus or HPV?"

Participants with a history of receptive anal sex and colorectal cancer were matched. The relative risk ratio was calculated using chi square test. Statistical analysis was conducted using the statistical software, R 3.5.1. Code available at: https://github.com/vincentmajor/ NHANES_receptive_anal_sex_in_women

\section{Results}

A total of 7,781 women completed the NHANES SXQ between 2009 and 2014 but only 6,596 answered question SXQ706 about receptive anal intercourse. A total of 2,203 women (28.3\%), with mean age of 42.4years (lower and upper quartiles: 29, 55) stated that they had a history of receptive anal intercourse at least once (Table 1). Within each age range, women between age 30-39 had the highest proportion of women with reported history of anal sex (43.1\%) whereas reported proportions were less for age ranges 50-59 (30.75) and 60-69 (23.2\%) (Figure 1). The most represented race/ethnicity was non-Hispanic white $(1065,48.3 \%)$ followed by non-Hispanic black (394, 17.9\%), and Mexican-American (309, 14.0\%). The rate of reported history of receptive anal intercourse did not significantly increase across the three cycles: $2009-2010(789,29.0 \%)$ to $2013-$ $2014(782,29.6 \%)$.

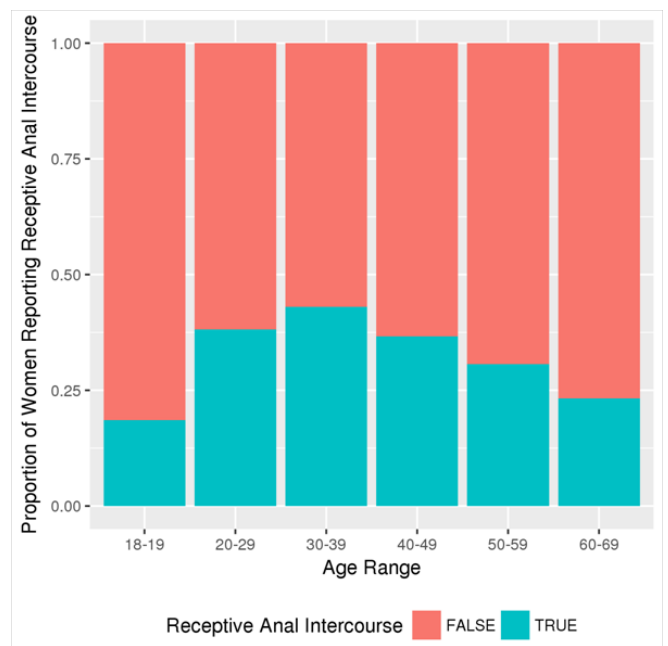

Figure I Age distribution of women reporting history or no history of anal sex . 
Table I Demographics of women reporting receptive anal sex $(n=2203)$

\begin{tabular}{|c|c|c|}
\hline Demographic & & Receptive Anal Sex n(\%) \\
\hline \multicolumn{3}{|l|}{ Age } \\
\hline & $18-19$ & $78(3.54)$ \\
\hline & $20-29$ & $488(22.2)$ \\
\hline & $30-39$ & $512(23.2)$ \\
\hline & $40-49$ & $478(21.7)$ \\
\hline & $50-59$ & $366(16.6)$ \\
\hline & $60-69$ & $28 I(12.7)$ \\
\hline \multicolumn{3}{|l|}{ Ethnicity } \\
\hline & Mexican American & $309(14.0)$ \\
\hline & Non-Hispanic Black & $394(17.9)$ \\
\hline & Non-Hispanic White & $1065(48.3)$ \\
\hline & Other Hispanic & $249(11.3)$ \\
\hline & $\begin{array}{l}\text { Other Race - Including } \\
\text { Multi-Racial }\end{array}$ & $186(8.4)$ \\
\hline
\end{tabular}

Of those women who answered SXQ706 between 2009 to 2014 , there were a total of 19 participants $(0.29 \%)$ with a diagnosis of colorectal cancer, with 5 patients who had both a diagnosis of colorectal cancer and history of anal intercourse. The risk of a diagnosis of colorectal cancer with a history of receptive anal intercourse in women was 0.0023 , whereas the risk of a diagnosis of colorectal cancer with no history of receptive anal intercourse was 0.0032 (Table 2). This yields a relative risk (RR) of 0.72 with chisquare $\mathrm{p}$-value $=0.68$. There was no significant evidence that receptive anal intercourse in women is associated with risk of colorectal cancer

Table 2 History of receptive anal sex in women with colorectal cancer diagnosis

\begin{tabular}{llll}
\hline & $\begin{array}{l}\text { History of } \\
\text { colorectal } \\
\text { cancer }\end{array}$ & $\begin{array}{l}\text { No history of } \\
\text { colorectal cancer }\end{array}$ & $\begin{array}{l}\text { Relative } \\
\text { risk }\end{array}$ \\
\hline $\begin{array}{l}\text { History of } \\
\text { receptive } \\
\text { anal sex }\end{array}$ & 5 & 2198 & 0.0023 \\
$\begin{array}{l}\text { No history } \\
\text { of receptive } \\
\text { anal sex }\end{array}$ & 14 & 4379 & 0.0032 \\
\hline
\end{tabular}

There were insufficient data for concurrent HPV infection and colorectal cancer in those participants without a history of anal sex, thus we could not assess the role of HPV in the association between receptive anal sex and CRC diagnosis. There is a significant association between receptive anal sex and HPV status in women $(\mathrm{RR}=1.64$ with $\mathrm{p}<0.0001)$, indicating women with HPV infection were 1.64 times more likely to report a history of anal sex than women with no reported HPV.

\section{Discussion}

Our findings suggest that a significant proportion of women have engaged in receptive anal sex across age groups and ethnicities. In this study, we found no significant association between receptive anal sex and CRC. Although prior hypotheses had suggested that altered microbiome or epithelial injury from lubricants could lead to increased risk of $\mathrm{CRC}$, our results did not confirm these hypotheses.

Results from the NHANES questionnaire did not suggest an increasing trend of receptive anal intercourse from 2009 to 2014. This differs from general population surveys in the United States that have suggested that the prevalence of anal intercourse among heterosexuals increased over time since the $1990 \mathrm{~s} .{ }^{23-25}$ These time trends were recorded over a longer time period of 10 to 20years, whereas our study compared incidence over a short period of only five years.

HPV is a known oncogenic virus, which is commonly spread via unprotected anal sex. ${ }^{16}$ Unfortunately, we were not able to test for a difference between risk of CRC for women with HPV as compared to women without HPV given insufficient sample size of HPV positive, CRC positive participants in the NHANES database. This is an important area of future study as recent studies have suggested that the HPV virus could be involved in the pathogenesis of CRC. ${ }^{16,17,19,21}$ One meta-analysis by Baandrup et al. concluded that HPV may be associated with a subset of colorectal cancers and there are also studies which physiologically link HPV to CRC tumors. ${ }^{26,27}$

The strength of our study is the utilization of a large, national survey that collects data from diverse populations which addresses the specific sexual behavior of receptive anal sex in women. Although questionnaires regarding sexual behavior can be difficult to obtain, this survey was able to identify several aspects of comprehensive sexual health among an extensive number of patients from diverse geographic locations in the United States.

The present analysis is not without limitations. First, history of receptive anal sex and colorectal cancer was self-reported and therefore subject to reporting bias. Second, in this cohort, the number of respondents with a diagnosis of $\mathrm{CRC}$ was small, $\mathrm{n}=19$ (prevalence of CRC in study population $=0.29 \%$ ), though this is similar to known prevalence of CRC in the United States, $0.41 \%$, assuming 2015 population of $321,000,000 .{ }^{28}$ Also, the questionnaire regarding male participants is inadequate and does not distinguish between insertive and receptive anal sex and thus could not be included in the present analysis. Future iterations of the NHANES SXQ should appropriately distinguish these sexual behaviors, so that the risk of colorectal cancer can be assessed in men. In future studies, it may be effective to retrospectively analyze a cohort of patients with CRC to look at their history and frequency of anal sex in comparison to a control cohort. To more adequately examine the interaction between anal sex and CRC incidence, more data of a higher quality measuring frequency of anal intercourse, specifically with respect to insertive versus receptive practices, is needed. Additional longitudinal studies evaluating the role of HPV in colorectal carcinogenesis are needed to evaluate HPV as a causative agent in CRC.

\section{Conclusion}

A significant proportion of women reported a history of receptive anal sex. Results from the NHANES questionnaire did not suggest an increasing trend of receptive anal intercourse from 2009 to 2014. No significant difference was found in relative risk of diagnosis of colorectal cancer in women who engaged in receptive anal intercourse as compared to those who did not engage in anal intercourse. We were unable to assess mediation or confounding by HPV infection. 


\section{Acknowledgments}

None.

\section{Conflict of interest}

The authors declare that there is no conflict of interest.

\section{Funding}

None.

\section{References}

1. American Cancer Society. Cancer Facts and Figures 2019. 2019

2. Siegel RL, Miller KD, Fedewa SA, et al. Colorectal cancer statistics, 2017. CA Cancer J Clin. 2017;67(3):177-193.

3. Campos FG. Colorectal cancer in young adults: a difficult challenge. World J Gastroenterol. 2017;23(28):5041.

4. Ambe PC, Gödde D, Störkel S, et al. Extra nodular metastasis is a poor prognostic factor for overall survival in node-positive patients with colorectal cancer. Int J Colorectal Dis. 2018;33(4):403-409.

5. Crosbie AB, Roche LM, Johnson LM, et al. Trends in colorectal cancer incidence among younger adults - Disparities by age, sex, race, ethnicity, and subsite. Cancer Med. 2018;7(8):4077-4086.

6. Waite LJ, Laumann EO, Das A, et al. Sexuality: Measures of partnerships, practices, attitudes, and problems in the National Social Life, Health, and Aging Study. J Gerontol B Psychol Sci Soc Sci. 2009;64(suppl_1):i56i66.

7. McBride KR, Fortenberry JD. Heterosexual anal sexuality and anal sex behaviors: a review. J Sex Res. 2010;47(2-3):123-136.

8. Kanouse DE, Berry SH, Gorman EM, et al. Response to the AIDS Epidemic. A Survey of Homosexual and Bisexual Men in Los Angeles County. Santa Monica, USA: RAND; 1991. pp. 1-112.

9. Owen BN, Brock PM, Butler AR, et al. Prevalence and frequency of heterosexual anal intercourse among young people: a systematic review and meta-anaysis. AIDS Behav. 2015;19(7):1338-1360.

10. Halperin DT. Heterosexual anal intercourse: prevalence, cultural factors, and HIV infection and other health risks, Part I. AIDS Patient Care STDS 1999;13(12):717-730.

11. Borovkova N, Korrovits P, Ausmees K, et al. Influence of sexua intercourse on genital tract microbiota in infertile couples. Anaerobe. $2011 ; 17(6): 414-418$

12. Kelley CF, Kraft CS, de Man TJ, et al. The rectal mucosa and condomless receptive anal intercourse in HIV-negative MSM: implications for HIV transmission and prevention. Mucosal Immunol. 2017;10(4):996.

13. Flemer B, Lynch DB, Brown JM, et al. Tumour-associated and nontumour-associated microbiota in colorectal cancer. Gut. 2017;66(4):633643.
14. Kasai C, Sugimoto K, Moritani I, et al. Comparison of human gut microbiota in control subjects and patients with colorectal carcinoma in adenoma: Terminal restriction fragment length polymorphism and nextgeneration sequencing analyses. Oncol Rep. 2016;35(1):325-333.

15. Sobhani I, Tap J, Roudot-Thoraval F, et al. Microbial dysbiosis in colorectal cancer (CRC) patients. PLoS One. 2011;6(1):e16393.

16. Lin C, Franceschi S, Clifford GM. Human papillomavirus types from infection to cancer in the anus, according to sex and HIV status: a systematic review and meta-analysis. Lancet Infect Dis. 2018;18(2):198206.

17. Bodaghi S, Yamanegi K, Xiao SY, et al. Colorectal papillomavirus infection in patients with colorectal cancer. Clin Cancer Res. 2005;11(8):2862-2867.

18. Damin DC, Caetano MB, Rosito MA, et al. Evidence for an association of human papillomavirus infection and colorectal cancer. Eur J Surg Oncol. 2007;33(5):569-574.

19. Bernabe-Dones RD, Gonzalez-Pons M, Villar-Prados A, et al. High prevalence of human papillomavirus in colorectal cancer in Hispanics: a case-control study. Gastroenterol Res Pract. 2016;2016:7896716.

20. Lee YM, Leu SY, Chiang H, et al. Human papillomavirus type 18 in colorectal cancer. J Microbiol Immunol Infect. 2001;34(2):87-91.

21. Vuitton L, Jaillet C, Jacquin E, et al. Human papillomaviruses in colorectal cancers: A case-control study in western patients. Dig Liver Dis. 2017;49(4):446-450.

22. Fuchs EJ, Lee LA, Torbenson MS, et al. Hyperosmolar sexual lubrican causes epithelial damage in the distal colon: potential implication for HIV transmission. J Infect Dis. 2007;195(5):703-710.

23. Aral SO, Patel DA, Holmes KK, et al. Temporal trends in sexual behaviors and sexually transmitted disease history among 18-to 39year-old Seattle, Washington, residents: results of random digit-dial surveys. Sex Transm Dis. 2005;32(11):710-717.

24. Mosher WD, Chandra A, Jones J. Sexual behavior and selected health measures: men and women 15-44 years of age, United States, 2002. Adv Data. 2005;(362):1-55

25. Leichliter JS, Chandra A, Liddon N, et al. Prevalence and correlates of heterosexual anal and oral sex in adolescents and adults in the United States. J Infect Dis. 2007;196(12):1852-1859.

26. Baandrup L, Thomsen LT1 Olesen TB, et al. The prevalence of human papillomavirus in colorectal adenomas and adenocarcinomas: a systematic review and meta-analysis. Eur J Cancer. 2014;50(8):1446-1461.

27. Siegel RL, Ward EM, Jemal A. Trends in colorectal cancer incidence rates in the United States by tumor location and stage, 1992-2008. Cancer Epidemiol Biomarkers Prev. 2012;21(3):411-416.

28. Institute NC. Cancer Stat Facts: Colorectal Cancer. Surveillance Research Program (SRP). 2015. 